\title{
Altered Consciousness and Atrial Fibrillation due to Primidone Intake
}

\author{
Ataman Köse', Seyran Bozkurt ${ }^{1}$, Ahmet Celik ${ }^{2}$, İpek Ağar ${ }^{1}$, Hüseyin Narcı', Cüneyt Ayrık \\ 'Department of Emergency Medicine, Mersin University Faculty of Medicine, Mersin, Turkey \\ ${ }^{2}$ Department of Cardiology, Mersin University Faculty of Medicine, Mersin, Turkey
}

\begin{abstract}
Primidone is an anticonvulsant drug taken for the treatment of essential tremor. Side effects may become more pronounced with increasing doses. However, atrial fibrillation as a side effect of the drug has neither been reported in literature nor is it specifically indicated by the package insert. Here we would like to report a patient who developed sedation, confusion, and atrial fibrillation after primidone use. A 68-year-old woman presented to our emergency department with nausea, vomiting, perspiration, and somnolence some time after taking primidone (Mysoline) $250 \mathrm{mg}$. It was learnt that Mysoline $62.5 \mathrm{mg}$ (a quarter of the $250 \mathrm{mg}$ tablet) had been prescribed by a neurologist for treating essential tremor 2-3 weeks before, although the patient had inadvertently taken a tablet per day. An admission electrocardiography (ECG) at the emergency department showed atrial fibrillation with a rapid ventricular response. The cardiology and neurology departments agreed that the atrial fibrillation episode was a side effect of primidone. It is recommended to instruct patients on and warn them against the side effects when primidone is taken for the first time. It should be kept in mind that in elderly patients presenting to emergency departments, drugs such as primidone, which may cause sedation, confusion, and atrial fibrillation, may be the cause. (Eurasian J Emerg Med 2015; 14: 151-3)
\end{abstract}

Keywords: Primidone, atrial fibrillation, sedation, emergency department

\section{Introduction}

Drug use and associated complications are common in the elderly. Multi-drug use, comorbid diseases, and dosing errors are important factors for the emergence of side effects. The inappropriate use of medications may be associated with increased rates of hospitalization, morbidity, mortality, and unexpected drug-related effects (1). Antiepileptic drugs are taken for a variety of indications in the elderly. Primidone is an anticonvulsant drug primarily taken for treating essential tremor. It is metabolized into phenobarbital and phenylethylmalonamide (PEMA). Its anticonvulsant actions are brought about by itself and its two active metabolites. However, its tremor suppression action is superior to that of its metabolites, and recently, it has been widely taken for essential tremor therapy $(2,3)$. Its side effects are common and generally dose dependent. The number and rate of side effects may become more prominent as the dose of the medication is increased $(4,5)$.
Depending on the dose of primidone, sedation, dizziness, and altered consciousness have been reported, although neither previous studies nor the package insert indicated that atrial fibrillation is among its side effects. In this study, we report a patient who experienced sedation, confusion, and atrial fibrillation after taking primidone.

\section{Case Presentation}

A 68-year-old woman presented to our emergency department with nausea, vomiting, perspiration, and somnolence after taking a primidone (Mysoline, Keymen Illaç San. and Tic. A.Ş, İstanbul, Turkey) $250 \mathrm{mg}$ tablet. Her past history was notable for hypertension and peripheral facial paralysis. She was prescribed a quarter of the primidone $250 \mathrm{mg}$ tablet per day by the neurology department for treating essential tremor 2-3 weeks before. However, she took one tablet a day $(250 \mathrm{mg})$. She reported excessive sweating, nausea, palpitation, and vomiting, followed by altered consciousness and progressive 
somnolence after taking the drug. At the initial examination, her Glasgow coma score was 14, and her vital signs were stable. She had a body temperature of $36^{\circ} \mathrm{C}$, pulse rate of $145 \mathrm{bpm}$, blood pressure of $140 / 90 \mathrm{mmHg}$, respiratory rate of 20 per min, and oxygen saturation of $94 \%$ in room air on pulse oximetry. The patient was monitored; a peripheral intravenous (IV) access was established, oxygen was administered, and blood samples were taken. Surface ECG showed atrial fibrillation with rapid ventricular response (Figure 1). As she had no history of heart or rhythm problems, she was considered to have new-onset atrial fibrillation and was given an anticoagulant as well as $25 \mathrm{mg}$ diltiazem for ventricular rate control, although diltiazem could not adequately control the ventricular rate. She was thus transferred to the cardiology department for the management of her atrial fibrillation and that department recommended amiodarone 150 $\mathrm{mg}$ infused in $100 \mathrm{cc}$ isotonic saline in $20 \mathrm{~min}$. Atrial fibrillation was converted to sinus rhythm after the amiodarone loading infusion (IV 150 mg; Cordarone ${ }^{\circledR}$ Sanofi Aventis, İstanbul, Turkey) in $100 \mathrm{~mL} 5 \%$ dextrose in $15 \mathrm{~min}$ ) (Figure 2). The echocardiogram performed after the restoration of her rhythm was normal. Her subsequent treatment regimen was adjusted by the cardiology department. Laboratory tests were all normal. The phenobarbital level was less than $0.5 \mathrm{mg} /$ $\mathrm{dL}$ and was normal. On neurological examination, the patient was somnolent and confused, in spite of the fact that her speech and understanding were normal. Muscle strength and sensory and reflex functions were also normal. She had no lateralizing muscle deficit. She was transferred to the neurology department for altered consciousness and somnolence. The neurology and cardiology departments jointly considered that the clinical condition of the patient

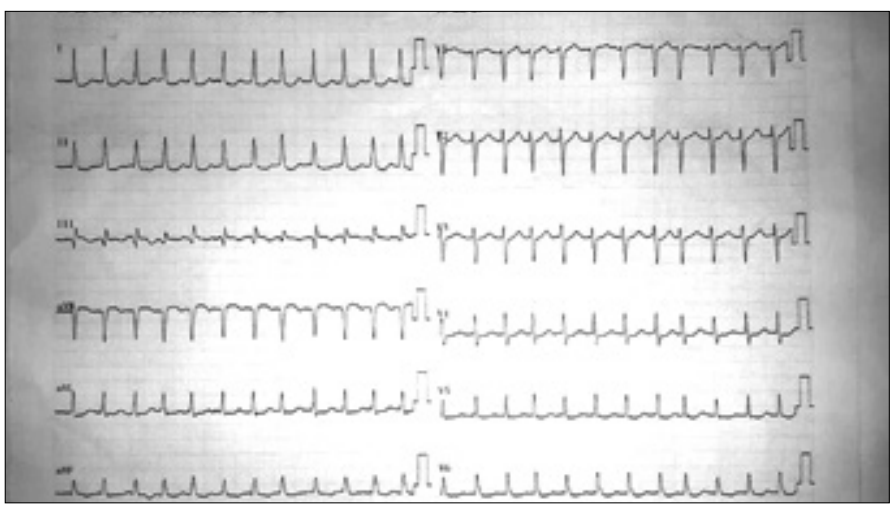

Figure 1. Atrial fibrillation in the initial ECG

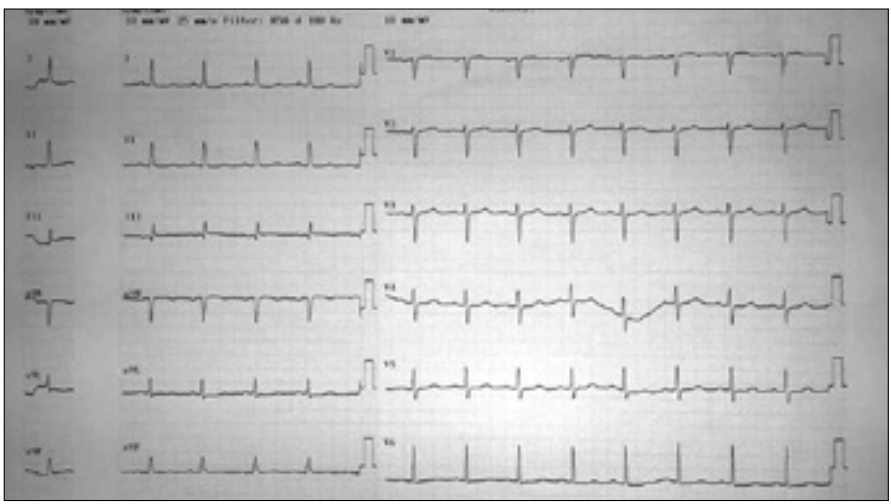

Figure 2. The rhythm converted to normal sinus rhythm after amiodarone infusion was a primidone-associated side effect. The patient's mental status and cardiac rhythm were monitored at the emergency department. She was administered IV fluids and gastric protection drugs. No further dysrhythmia occurred during the 36-h emergency department stay. Her mental status also improved later, and she was discharged upon the recommendations of the neurology and cardiology departments.

\section{Discussion}

Altered consciousness and rhythm problems may occur following the intake of many medications or substances. The above complications may either indicate drug overdose or side effects. Antiepileptics (particularly phenytoin and phenobarbital) may also cause these types of adverse reactions $(3,6)$. Primidone is a powerful central nervous system depressant. During the early stages of treatment, its side effects usually appear in the form of fatigue, somnolence, and confusion. Depending on the ingested dose, excessive dosing may cause varying levels of central nervous system depression, which includes ataxia, loss of consciousness, respiratory depression, and coma $(3,5)$. Some patients, particularly the elderly, may complain of confusion, dizziness, sedation, ataxia, nausea, and fatigue even after doses of $50 \mathrm{mg} /$ day or lower. The number and rate of side effects may become more prominent as the dose of the medication is increased. Thus, primidone should be started at very low doses (i.e. $25 \mathrm{mg}$ or even $12.5 \mathrm{mg}$ ) and half a quarter of the tablet it should be taken at bedtime to avoid side effects (dizziness and sedation) (3-5). Then, the dose can be increased to $250 \mathrm{mg}$ every third day, until the symptoms are controlled or the maximum tolerated dose is reached in adults. Our patient took one tablet at a time despite being advised to take a quarter of the $250 \mathrm{mg}$ tablet. She later presented with nausea, vomiting, dizziness, and somnolence. This clinical picture was consistent with the primidone side effect that was reported by both literature data and the primidone package insert.

Katano et al. (7) reported a case of hyperammonemic encephalopathy with impaired consciousness after primidone intake. The patient had elevated ammonia and phenobarbital levels despite normal routine laboratory test results. Unlike our patient, that patient also had an intracranial tumor. Our patient developed the clinical picture without concurrent primidone intoxication. The clinical picture improved upon the cessation of primidone. Koch et al. (8) observed that primidone-induced sedation may augment altered consciousness in the elderly, particularly in those with dementia. Thus, they recommended that a repeat examination be performed at regular intervals and that non-sedative antiepileptic agents be prescribed in such conditions.

Some antiepileptic drugs (phenobarbiturates, phenytoin) have been shown to induce cardiac arrhythmias in a dose-dependent manner (6). Potassium, sodium, and calcium ion channels are central to cardiac rhythm generation (9). Although the upregulation of the GABA-A receptors plays a vital role for the emergence of the effects of phenobarbital and primidone, these agents may also act through the inhibition of sodium, potassium, and calcium ion channels. The latter have been implicated in primidone's anticonvulsant and antitremor activities $(6,10,11)$. Hence, it has been suggested that taking these drugs in inappropriate doses poses an arrhythmia risk associated with the suppression of the ion channels. Some studies have reported that primidone is not an arrhythmogenic agent (12) but 
rather is an anticonvulsant agent with cardiac arrhythmia correction and QT interval shortening effects (13). Nevertheless, it caused atrial fibrillation in our patient, and this rhythm disorder may be related to the suppression of the conduction system.

The actions of primidone are mediated by itself and its two active metabolites (phenobarbital and PEMA). Thus, the phenobarbital level is usually checked in primidone toxicity. Because the phenobarbital level was normal and the intake did not occur at a very high dose, toxicity was not the case in our patient. Depending on the dose taken, the side effects may have appeared because of the advanced patient age and high serum drug concentrations. Compared with primidone, the serum levels of phenobarbital and PEMA tend to be higher. Renal clearance of all compounds (primidone, PEMA, and phenobarbital) is moderately impaired in the elderly, which is possibly related to reduced renal function with aging (2).

\section{Conclusion}

This case report suggests that primidone has a sedative and an arrhythmogenic potential. Patients who are prescribed primidone should be instructed on the drug's usage and warned about its side effects. Primidone should be considered in the differential diagnosis of new-onset atrial fibrillation, especially in the elderly.

Informed Consent: The patient consent was obtained as verbal from patients' relatives. In addition, it was not taken as the picture of patient does not include, and blood tests was taken for routine use.

Peer-review: Externally peer-reviewed.

Conflict of Interest: No conflict of interest was declared by the authors.

Financial Disclosure: The authors declared that this study has received no financial support.

\section{References}

1. Rahimi AR. Primidone and liver abnormalities: A case study. J Pharmacol Clin Toxicol 2014; 2: 1019.

2. Martines C, Gatti G, Sasso E, Calzetti S, Perucca E. The disposition of primidone in elderly patients. Br J Clin Pharmacol 1990; 30:607-11. [CrossRef]

3. Hedera P, Cibulčík F, Davis TL. Pharmacotherapy of essential tremor. J Cent Nerv Syst Dis 2013; 5: 43-55. [CrossRef]

4. Deuschl G, Raethjen J, Hellriegel H, Elble R. Treatment of patients with essential tremor. Lancet Neurol 2011; 10: 148-61. [CrossRef]

5. O'Suilleabhain P, Dewey RB Jr. Randomized trial comparing primidone initiation schedules for treating essential tremor. Mov Disord 2002; 17: 382-6. [CrossRef]

6. Danielsson BR, Lansdell K, Patmore L, Tomson T. Phenytoin and phenobarbital inhibit human HERG potassium channels. Epilepsy Res 2003; 55: 147-57. [CrossRef]

7. Katano H, Fukushima T, Karasawa K, Sugiyama N, Ohkura A, Kamiya K. Primidone-induced hyperammonemic encephalopathy in a patient with cerebral astrocytoma. J Clin Neurosci 2002; 9: 79-81. [CrossRef]

8. Koch HJ, Szecsey A, Vogel M. Sedation caused by primidone may exacerbate dementia. Epilepsy Behav 2003; 4: 592. [CrossRef]

9. Wang L, Feng ZP, Kondo CS, Sheldon RS, Duff HJ. Developmental changes in the delayed rectifier K+ channels in mouse heart. Circ Res 1996; 79: 79-85. [CrossRef]

10. Guan XM, Peroutka SJ. Basic mechanisms of action of drugs used in the treatment of essential tremor. Clin Neuropharmacol 1990; 13: 210-23. [CrossRef]

11. Rogawski MA, Porter RJ, Antiepileptic drugs: pharmacological mechanisms and clinical efficacy with consideration of promising developmental stage compounds. Pharmacol Rev 1990; 42: 223-86.

12. Stollberger $C$, Finsterer J. Cardiorespiratory findings in sudden unexplained/unexpected death in epilepsy (SUDEP). Epilepsy Res 2004; 59: 51-60. [CrossRef]

13. Christidis D, Kalogerakis D, Chan TY, Mauri D, Alexiou G, Terzoudi A. Is primidone the drug of choice for epileptic patients with QT-prolongation? A comprehensive analysis of literature. Seizure 2006; 15: 64-6. [CrossRef] 